\title{
Mutual Inductance and Magnetic Force Calculations for Bitter Disk Coil (Pancake) with Nonlinear Radial Current and Filamentary Circular Coil with Azimuthal Current
}

\author{
Slobodan Babic ${ }^{1}$ and Cevdet Akyel ${ }^{2}$ \\ ${ }^{1}$ Département de Génie Physique, École Polytechnique, CP 6079, Succ. Centre Ville, Montréal, QC, Canada H3C 3A7 \\ ${ }^{2}$ Département de Génie Électrique, École Polytechnique, CP 6079, Succ. Centre Ville, Montréal, QC, Canada H3C 3A7 \\ Correspondence should be addressed to Slobodan Babic; slobodan.babic@polymtl.ca
}

Received 12 June 2016; Revised 8 August 2016; Accepted 22 August 2016

Academic Editor: Gorazd Stumberger

Copyright (C) 2016 S. Babic and C. Akyel. This is an open access article distributed under the Creative Commons Attribution License, which permits unrestricted use, distribution, and reproduction in any medium, provided the original work is properly cited.

Bitter coils are electromagnets used for the generation of extremely strong magnetic fields superior to $30 \mathrm{~T}$. In this paper we calculate the mutual inductance and the magnetic force between Bitter disk (pancake) coil with the nonlinear radial current and the circular filamentary coil with the azimuthal current. The close form expressed over complete elliptic integrals of the first and second kind as well as Heuman's Lambda function is obtained for this configuration either for the mutual inductance or for the magnetic force. The results of this method are compared with those obtained by the improved modified filament method for the presented configuration. All results are in an excellent agreement.

\section{Introduction}

In the literature and scientific papers the calculation of the mutual inductance and the magnetic force between ordinary circular coils with the uniform current densities have been given by different methods either analytical or numerical [1-15]. However, there are not a lot references about the mutual inductance and the magnetic force calculation between circular coils in which the current densities are not uniform. The coils with this characteristic are Bitter coils or Bitter solenoids. Bitter coils are used in high magnetic field applications and they differ from the ordinary coils in that they have an inverse radial distribution of current $[16,17]$. The interesting point is the calculation of the mutual inductance and the magnetic force between Bitter coils or between one Bitter coil and an ordinary coil. Generally, the calculation of the magnetic force between circular coils is closely related to the calculation of their mutual inductance. Since their mutual energy is equal to the product of their mutual inductance and the currents in the coils, the component of the magnetic force of attraction or repulsion in any direction is equal to the product of the currents multiplied by the differential coefficient of the mutual inductance taken with respect to that coordinate. The mutual inductance and the magnetic force are obtained over multiple integrals with the different kernel functions. Thus, in the calculation of the mutual inductance and the corresponding magnetic force between two coils we need to integrate their kernel functions depending on coil configurations. Hopefully, these kernel functions are Green functions $r^{-0.5}$ and $r^{-1.5}$, where the " $r$ " is the distance between two coils. This integral approach, much easier than the differential approach, leads to relatively simple expressions which are incorporated in these two physical quantities. In this paper we calculate the mutual inductance between the Bitter disk coil with nonlinear radial current and a circular filamentary coil with the azimuthal current (ordinary coil). Coils are coaxial and in air. Either the mutual inductance or the magnetic force is obtained in closed form expressed over complete elliptic integrals of the first and second kind as well as over Heuman's Lambda function $[18,19]$. The results of these calculations will be compared to those obtained by the improved filament method for concerned configuration. The results obtained by these two methods are in an excellent agreement. 

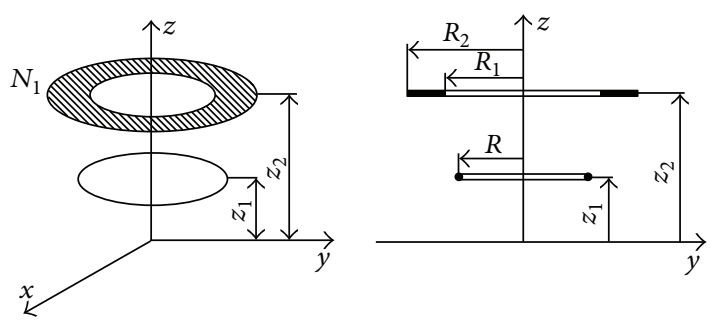

Figure 1: The thin Bitter disk coil (pancake) and the filamentary circular coil.

\section{Basic Expressions}

The mutual inductance and magnetic force between a disk coil (pancake) with the uniform azimuthal current density $J_{1}=N_{1} I_{1} /\left(R_{2}-R_{1}\right)$ and a filamentary coil with the azimuthal current $I_{2}$ (see Figure 1) can be calculated, respectively, by $[1,2]$

$$
\begin{aligned}
M & =\frac{\mu_{0} N_{1} R}{\left(R_{2}-R_{1}\right)} \int_{0}^{\pi} \int_{R_{1}}^{R_{2}} \frac{r_{I} \cos \theta d r_{I} d \theta}{r_{0}}, \\
F & =-\frac{\mu_{0} N_{1} I_{1} I_{2} R\left(z_{2}-z_{1}\right)}{\left(R_{2}-R_{1}\right)} \int_{0}^{\pi} \int_{R_{1}}^{R_{2}} \frac{r_{I} \cos \theta d r_{I} d \theta}{r_{0}^{3}},
\end{aligned}
$$

where

$$
r_{0}=\sqrt{\left(z_{2}-z_{1}\right)^{2}+r_{I}^{2}+R^{2}-2 r_{I} R \cos \theta}
$$

and $N_{1}$ is the number of turns of the disk coil (pancake).

$\mu_{0}=4 \pi \times 10^{-7} \mathrm{H} / \mathrm{m}$ is the permeability of free space (vacuum), and $r, \theta, z$ are the cylindrical coordinates. Both coils are in air or in a nonmagnetic and nonconducting environment.

In [2] the magnetic force is obtained in the close form expressed over the complete elliptic integral of the first kind and Heuman's Lambda function and the magnetic force in the semianalytical form expressed over complete elliptic integral of the first and second kind, Heuman's Lambda function plus one simple integral which has to be evaluated by some of the numerical integrations [1].

Let us suppose the disk coil be the Bitter coil in which the current density is not uniform $[16,17]$ and given by

$$
J_{1}=\frac{N_{1} I_{1}}{r_{I} \ln \left(R_{2} / R_{1}\right)} .
$$

From (1), (2), and (4) we obtain the expressions of the mutual inductance and the magnetic force between the Bitter coil and the filamentary coil as follows:

$$
\begin{aligned}
M_{B} & =\frac{\mu_{0} N_{1} R}{\ln \left(R_{2} / R_{1}\right)} \int_{0}^{\pi} \int_{R_{1}}^{R_{2}} \frac{\cos \theta d r_{I} d \theta}{r_{0}}, \\
F_{B} & =-\frac{\mu_{0} N_{1} I_{1} I_{2} R\left(z_{2}-z_{1}\right)}{\ln \left(R_{2} / R_{1}\right)} \int_{0}^{\pi} \int_{R_{1}}^{R_{2}} \frac{\cos \theta d r_{I} d \theta}{r_{0}^{3}},
\end{aligned}
$$

where

$$
r_{0}=\sqrt{\left(z_{2}-z_{1}\right)^{2}+r_{I}^{2}+R^{2}-2 r_{I} R \cos \theta} .
$$

\section{Calculation Method}

Integrating in (5) and (6) over $r_{I}$ and $\theta$ (substituting $\theta=\pi-\beta$ ) the mutual inductance and the magnetic force between the Bitter disk and the filamentary circular coil can be expressed, respectively, in an analytical form as follow (see Appendices $\mathrm{A}$ and $\mathrm{B})$ :

$$
\begin{gathered}
M_{B}=\frac{\mu_{0} N_{1}}{2 \ln \left(R_{2} / R_{1}\right)} \sum_{n=1}^{n=2}(-1)^{n-1} T_{n}, \\
F_{B}=\frac{\mu_{0} N_{1} I_{1} I_{2}}{\ln \left(R_{2} / R_{1}\right)} \sum_{n=1}^{n=2}(-1)^{n-1} S_{n},
\end{gathered}
$$

where

$$
\begin{aligned}
T_{n}= & \frac{k_{n}}{\sqrt{R R_{n}}}\left[2 R \sqrt{R^{2}+z_{\mathrm{Q}}^{2}}-R_{n}^{2}-R^{2}-z_{\mathrm{Q}}^{2}\right] K\left(k_{n}\right) \\
& +\frac{k_{n}}{\sqrt{R R_{n}}}\left[\left(R+R_{n}\right)^{2}+z_{\mathrm{Q}}^{2}\right] E\left(k_{n}\right)-\pi\left|z_{\mathrm{Q}}\right| V_{n}, \\
S_{n}= & \frac{k_{n} z_{\mathrm{Q}}}{\sqrt{R R_{n}}} \frac{R}{\sqrt{R^{2}+z_{\mathrm{Q}}^{2}}+R} K\left(k_{n}\right)-\frac{\pi}{2} \operatorname{sgn}\left(z_{\mathrm{Q}}\right) V_{n}, \\
V_{n}= & 1-\Lambda_{0}\left(\theta_{1 n}, k_{n}\right) \\
& +\operatorname{sgn}\left(\sqrt{R^{2}+z_{\mathrm{Q}}^{2}}-R_{n}\right)\left[1-\Lambda_{0}\left(\theta_{2 n}, k_{n}\right)\right], \\
z_{\mathrm{Q}}= & z_{2}-z_{1}, \\
k_{n}^{2}= & \frac{4 R R_{n}}{\left(R+R_{n}\right)^{2}+z_{\mathrm{Q}}^{2}} \leq 1, \\
\theta_{2 n}= & \arcsin \sqrt{\frac{1-m}{1-k_{n}^{2}}, \quad k_{n}^{2} \leq m .} \\
m= & \frac{2 R}{\sqrt{R+z_{\mathrm{Q}}^{2}+R} \leq 1,} \\
& \arcsin \frac{\left|z_{\mathrm{Q}}\right|}{R^{2}+z_{\mathrm{Q}}^{2}}+R
\end{aligned}
$$

Thus, all expressions are obtained in the closed form expressed over complete elliptic integrals of the first and second kind as well as Heuman's Lambda function [18, 19].

\subsection{Special Cases and Singularities}

Mutual Inductance. In the mutual inductance calculation special cases and singular cases appear for $z_{Q}=0$ and $k_{n}^{2} \neq 1$ or $z_{\mathrm{Q}}=0$ and $k_{n}^{2}=1$. 
3.1.1. $z_{\mathrm{Q}}=0$ and $k_{n}^{2} \neq 1$. This case is the regular case covered by (8). In this case the term $T_{n}$ can be given in the following simplified form:

$$
\begin{aligned}
T_{n} & =\frac{k_{n 0}\left(R+R_{n}\right)}{\sqrt{R R_{n}}}\left[\left(R-R_{n}\right) K\left(k_{n 0}\right)\right. \\
& \left.+\left(R+R_{n}\right) E\left(k_{n 0}\right)\right], \\
k_{n 0}^{2} & =\frac{4 R R_{n}}{\left(R+R_{n}\right)^{2}} \leq 1 .
\end{aligned}
$$

3.1.2. $z_{\mathrm{Q}}=0$ and $k_{n}^{2}=1$. This case is the singular case $(R=$ $R_{1}$ or $R=R_{2}$ ) for which the term $T_{n}$ is equal either $4 R_{1}$ or $4 R_{2}$.

Magnetic Force. In the magnetic force calculation for $z_{Q}=0$ its value is zero because the coils are in the same plane.

Notation Above. $\Lambda_{0}$ corresponds to Heuman's Lambda function as defined in [18, 19] and "sign" function returns integer indicating the sign of a number. Functions $K$ and $E$ correspond to complete elliptic integrals of the first and second kind $[18,19]$.

\section{Modified Filament Method}

In [17] the mutual inductance is calculated between the Bitter coil and the superconducting coil with uniform current density by using the filament method. Here we give the modified formulas for the mutual inductance and the magnetic force for the treated configuration (see Figure 2) using the filament method.

Applying some modification in the mutual inductance calculation given in [17] we deduced the mutual inductance and the magnetic force between the Bitter coil and the filamentary coil as follows:

$$
\begin{gathered}
M_{\mathrm{BF}}=\frac{N_{1}\left(R_{2}-R_{1}\right)}{(2 n+1) \ln \left(R_{2} / R_{1}\right)} \sum_{l=-n}^{l=n} \frac{M(l)}{r_{I}(l)}, \\
F_{\mathrm{BF}}=\frac{N_{1} I_{1} I_{2}\left(R_{2}-R_{1}\right)}{(2 n+1) \ln \left(R_{2} / R_{1}\right)} \sum_{l=-n}^{l=n} \frac{F(l)}{r_{I}(l)},
\end{gathered}
$$

where

$$
\begin{aligned}
& M(l) \\
& \quad=\frac{2 \mu_{0} \sqrt{r_{I}(l) R}}{k(l)}\left[\left(1-\frac{k^{2}(l)}{2}\right) K(k(l))-E(k(l))\right],
\end{aligned}
$$$$
F(l)
$$$$
=-\frac{\mu_{0} I_{1} I_{2} c k(l)}{4 \sqrt{r_{I}(l) R}}\left[\frac{2-k^{2}(l)}{1-k^{2}(l)} E(k(l))-2 K(k(l))\right],
$$

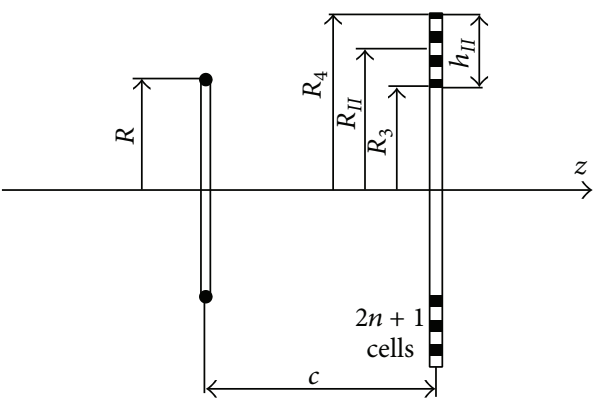

FIGURE 2: Configuration of mesh matrix: Filamentary coil-thin disk coil (pancake).

$$
\begin{aligned}
& r_{I}(l)=\frac{R_{2}+R_{1}}{2}+\frac{R_{2}-R_{1}}{2 n+1} l \quad(l=-n, \ldots, 0, \ldots, n), \\
& c=z_{2}-z_{1}=z_{Q} \\
& k^{2}(l)=\frac{4 r_{I}(l) R}{\left(r_{I}(l)+R\right)^{2}+c^{2}} .
\end{aligned}
$$

Expressions (13) will be used to confirm the validity of analytical formulas (8) and (9).

\section{Examples}

To verify the validity of the new formulas, we apply them to the following set of examples.

Example 1. Calculate the mutual inductance and the magnetic force between the Bitter disk coil and a filamentary circular coil with the following dimensions and the number of turns. Currents in coils are unit.

Filamentary Circular Coil. $R=20 \mathrm{~mm}, z_{1}=0 \mathrm{~mm}$.

Bitter Disk Coil. $R_{1}=40 \mathrm{~mm}, R_{2}=60 \mathrm{~mm}, z_{2}=50 \mathrm{~mm}$, $N_{1}=100$.

Applying (8) and (9) the mutual inductance and the magnetic force are, respectively,

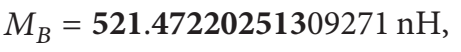

$$
\begin{aligned}
& F_{B}=-16.016662833788816 \mu \mathrm{N} \text {. }
\end{aligned}
$$

The computational time is about 0.037677 seconds.

By using the modified filament, (13) and (14), we obtain

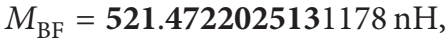

$$
\begin{aligned}
& F_{\mathrm{BF}}=-\mathbf{1 6 . 0 1 6 6 6 2 8 3 3 7 8 8 9 5 \mu \mathrm { N }} \text {. }
\end{aligned}
$$

The number of subdivision was $n=1000000$ and the computational time about 0.362069 seconds.

Example 2. Calculate the mutual inductance between a disk and a filamentary circular coil with the following dimensions and the number of turns. 
Filamentary Circular Coil. $R=20 \mathrm{~mm}, z_{1}=0 \mathrm{~mm}$.

Disk Coil. $R_{1}=40 \mathrm{~mm}, R_{2}=60 \mathrm{~mm}, z_{2}=0 \mathrm{~mm}, N_{1}=100$.

Applying (8) and (9) the mutual inductance and the magnetic force are, respectively,

$$
\begin{aligned}
M_{B} & =1.7442876932070914 \mu \mathrm{H}, \\
F_{B} & =0 \mathrm{~N} .
\end{aligned}
$$

The computational time is about 0.020657 seconds.

By using the modified filament, (13) and (14), we obtain

$$
\begin{aligned}
M_{\mathrm{BF}} & =1.744287693207058 \mu \mathrm{H}, \\
F_{\mathrm{BF}} & =0 \mathrm{~N} .
\end{aligned}
$$

The number of subdivision was $n=20000$ and the computational time about 0.030564 seconds.

Example 3. Calculate the mutual inductance between a disk and a filamentary circular coil with the following dimensions and the number of turns.

Filamentary Circular Coil. $R=40 \mathrm{~mm}, z_{1}=0 \mathrm{~mm}$.

Disk Coil. $R_{1}=40 \mathrm{~mm}, R_{2}=60 \mathrm{~mm}, z_{2}=0 \mathrm{~mm}, N_{1}=100$.

This case is the singular case because $R=R_{1}$.

Applying (8), (9), and (11) the mutual inductance and the magnetic force are, respectively,

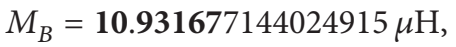

$$
\begin{aligned}
& F_{B}=0 \mathrm{~N} \text {. }
\end{aligned}
$$

The computational time is about 0.020223 seconds.

By using the modified filament, (13) and (14), we obtain

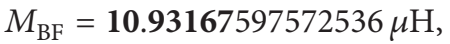

$$
\begin{aligned}
& F_{\mathrm{BF}}=0 \mathrm{~N} \text {. }
\end{aligned}
$$

The number of subdivision was $n=1000000$ and the computational time about 0.513017 seconds.

Example 4. Calculate the mutual inductance between a disk and a filamentary circular coil with the following dimensions and the number of turns.

Filamentary Circular Coil. $R=60 \mathrm{~mm}, z_{1}=0 \mathrm{~mm}$.

Disk Coil. $R_{1}=40 \mathrm{~mm}, R_{2}=60 \mathrm{~mm}, z_{2}=0 \mathrm{~mm}, N_{1}=100$.

This case is also the singular case for which $R=R_{2}$.

Applying (8), (9), and (11) the mutual inductance and the magnetic force are, respectively,

$$
\begin{aligned}
M_{B} & =14.062056002507577 \mu \mathrm{H}, \\
F_{B} & =0 \mathrm{~N} .
\end{aligned}
$$

The computational time is about 0.03929 seconds.
By using the modified filament, (13) and (14), we obtain

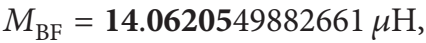

$$
\begin{aligned}
& F_{\mathrm{BF}}=0 \mathrm{~N} \text {. }
\end{aligned}
$$

The number of subdivision was $n=1000000$ and the computational time about 0.487595 seconds.

In comparative calculations the significant figures which agree are bolded.

Thus, with these examples we confirmed the validity of the present analytical method for calculating the mutual inductance and the magnetic force between the Bitter disk with the nonlinear radial current distribution and an ordinary filamentary circular coil. All calculations were executed in Matlab programming.

\section{Conclusion}

The new accurate mutual inductance and magnetic force formulas for the system of the Bitter disk with the nonlinear radial current and the filamentary circular with the azimuthal in air are derived and presented in this paper. All expressions for either the mutual inductance or the magnetic force are obtained in the close form expressed over complete elliptic integrals of the first and second kind and Heuman's Lambda function. Also we gave in this paper the improved formulas for the mutual inductance and the magnetic force between treated coils by using the filament method. All singular cases are obtained in a closed form. The presented method can be used to calculate these important electrical quantities such as the mutual inductance and the magnetic force for coils combinations comprising Bitter coils of rectangular cross section and the circular coils with uniform current densities.

\section{Appendix}

\section{A. Mutual Inductance Integrals}

The first integral in (5) is $I_{10}$ which can be solved analytically [19]:

$$
\begin{aligned}
I_{10} & =\int_{R_{1}}^{R_{2}} \frac{d r_{I}}{\sqrt{r_{I}^{2}+2 r_{I} R \cos 2 \beta+R^{2}+z_{Q}^{2}}} \\
& =\left.\operatorname{aresh} \frac{r_{I}+R \cos 2 \beta}{\sqrt{R^{2} \sin ^{2} 2 \beta+z_{Q}^{2}}}\right|_{R_{1}} ^{R_{2}} .
\end{aligned}
$$

The last integral in (5) is

$$
I_{20}=\int_{0}^{\pi / 2} \cos 2 \beta \cdot \operatorname{aresh} \frac{R_{n}+R \cos 2 \beta}{\sqrt{R^{2} \sin ^{2} 2 \beta+z_{Q}^{2}}} d \beta
$$


which can be obtained in the following form:

$$
I_{20}=\frac{k_{n}}{2} \sqrt{\frac{R_{n}}{R}} \int_{0}^{\pi / 2} \frac{\cos ^{3} 2 \beta+\left(\left(R^{2}+z_{\mathrm{Q}}^{2}\right) / R R_{n}\right) \cos ^{2} 2 \beta-\cos 2 \beta-\left(R^{2}+z_{\mathrm{Q}}^{2}\right) / R R_{n}}{\left(\cos ^{2} 2 \beta-\left(R^{2}+z_{\mathrm{Q}}^{2}\right) / R^{2}\right) \Delta} d \beta=\frac{k_{n}}{2} \sqrt{\frac{R_{n}}{R}} I_{0},
$$

where

$$
\Delta=\sqrt{1-k_{n}^{2} \sin ^{2} 2 \beta}
$$

$$
\begin{aligned}
& z_{\mathrm{Q}}=z_{2}-z_{1}, \\
& k_{n}^{2}=\frac{4 R R_{n}}{\left(R+R_{n}\right)^{2}+z_{\mathrm{Q}}^{2}} \leq 1,
\end{aligned}
$$

The integral $I_{0}$ can be obtained in its final form expressed over complete elliptic integrals of the first and second kind $K(k)$, $E(k)$ as well as Heuman's Lambda function $\Lambda_{0}(\varepsilon, k)[18,19]$ :

$$
\begin{aligned}
I_{0} & =\int_{0}^{\pi / 2} \frac{\cos ^{3} 2 \beta+\left(\left(R^{2}+z_{\mathrm{Q}}^{2}\right) / R R_{n}\right) \cos ^{2} 2 \beta-\cos 2 \beta-\left(R^{2}+z_{\mathrm{Q}}^{2}\right) / R R_{n}}{\left(\cos ^{2} 2 \beta-\left(R^{2}+z_{\mathrm{Q}}^{2}\right) / R^{2}\right) \Delta} d \beta=\int_{0}^{\pi / 2} \frac{\cos 2 \beta+\left(R^{2}+z_{\mathrm{Q}}^{2}\right) / R R_{n}}{\Delta} d \beta \\
& +\frac{z_{\mathrm{Q}}^{2}}{R^{2}} \int_{0}^{\pi / 2} \frac{\cos 2 \beta+\left(R^{2}+z_{\mathrm{Q}}^{2}\right) / R R_{n}}{\left(\cos ^{2} 2 \beta-\left(R^{2}+z_{\mathrm{Q}}^{2}\right) / R^{2}\right) \Delta} d \beta=\frac{2 R \sqrt{R^{2}+z_{\mathrm{Q}}^{2}}-R_{n}^{2}-R^{2}-z_{\mathrm{Q}}^{2}}{2 R R_{n}} K\left(k_{n}\right)+\frac{\left(R+R_{n}\right)^{2}+z_{\mathrm{Q}}^{2}}{2 R R_{n}} E\left(k_{n}\right) \\
& -\frac{\pi\left|z_{\mathrm{Q}}\right|}{2 k_{n} \sqrt{R R_{n}}} V_{n},
\end{aligned}
$$

where

$$
\begin{aligned}
V_{n}=1 & -\Lambda_{0}\left(\theta_{1 n}, k_{n}\right) \\
& +\operatorname{sgn}\left(\sqrt{R^{2}+z_{\mathrm{Q}}^{2}}-R_{n}\right)\left[1-\Lambda_{0}\left(\theta_{2 n}, k_{n}\right)\right] .
\end{aligned}
$$

Replacing $I_{20}$ in expression (5) we obtain (8).

\section{B. Magnetic Force Integrals}

The first integral in (6) is $L_{10}$ which has the analytic solution [19]:

$$
\begin{aligned}
& L_{10}=\int_{R_{1}}^{R_{2}} \frac{d r_{I}}{\left(r_{I}^{2}+2 r_{I} R \cos 2 \beta+R^{2}+z_{Q}^{2}\right)^{3 / 2}} \\
& =\left.\frac{r_{I}+R \cos 2 \beta}{\left(R^{2} \sin ^{2} 2 \beta+z_{Q}^{2}\right) \sqrt{r_{I}^{2}+2 r_{I} R \cos 2 \beta+R^{2}+z_{Q}^{2}}}\right|_{R_{1}} ^{R_{2}} .
\end{aligned}
$$

The last integral in (6) is

$$
\begin{aligned}
& L_{20} \\
& =2 R z_{\mathrm{Q}} \int_{0}^{\pi / 2} \frac{\cos 2 \beta\left(R_{n}+R \cos 2 \beta\right) d \beta}{\left(R^{2} \sin ^{2} 2 \beta+z_{\mathrm{Q}}^{2}\right) \sqrt{r_{I}^{2}+2 r_{I} R \cos 2 \beta+R^{2}+z_{\mathrm{Q}}^{2}}}
\end{aligned}
$$

which can be given in the following form:

$$
\begin{aligned}
L_{20} & =-\frac{k_{n} z_{\mathrm{Q}}}{\sqrt{R_{n} R}}\left[\int_{0}^{\pi / 2} \frac{d \beta}{\Delta}\right. \\
& \left.+\int_{0}^{\pi / 2} \frac{\cos 2 \beta+\left(R^{2}+z_{\mathrm{Q}}^{2}\right) / R R_{n}}{\left(\cos ^{2} 2 \beta-\left(R^{2}+z_{\mathrm{Q}}^{2}\right) / R^{2}\right) \Delta} d \beta\right] \\
& =-\frac{k_{n} z_{\mathrm{Q}}}{\sqrt{R_{n} R}}\left[\int_{0}^{\pi / 2} \frac{d \beta}{\Delta}+\frac{R_{n}}{R} I_{0}\right] \\
& =-\frac{k_{n} z_{\mathrm{Q}}}{\sqrt{R_{n} R}}\left[K\left(k_{n}\right)+\frac{R_{n}}{R} I_{0}\right] .
\end{aligned}
$$

Integral $I_{0}$ appears in Appendix A so that replacing $L_{20}$ in (6) we obtain (9).

\section{Competing Interests}

The authors declare that there is no conflict of interests regarding the publication of this paper.

\section{References}

[1] C. Akyel, S. I. Babic, and S. Kincic, "New and fast procedures for calculating the mutual inductance of coaxial circular coils (circular coil-disk coil)," IEEE Transactions on Magnetics, vol. 38, no. 5, pp. 2367-2369, 2002. 
[2] C. Akyel, S. I. Babic, S. Kincic, and P. J. Lagacé, "Magnetic force calculation between thin circular coils and thin filamentary circular coil in air," Journal of Electromagnetic Waves and Applications, vol. 21, no. 9, pp. 1273-1283, 2007.

[3] S. Babic, C. Akyel, Y. Ren, and W. Chen, "Magnetic force calculation between circular coils of rectangular cross section with parallel axes for superconducting magnet," Progress In Electromagnetics Research B, no. 37, pp. 275-288, 2012.

[4] S. I. Babic and C. Akyel, "New analytic-numerical solutions for the mutual inductance of two coaxial circular coils with rectangular cross section in air," IEEE Transactions on Magnetics, vol. 42, no. 6, pp. 1661-1669, 2006.

[5] S. I. Babic and C. Akyel, "Magnetic force calculation between thin coaxial circular coils in air," IEEE Transactions on Magnetics, vol. 44, no. 4, pp. 445-452, 2008.

[6] J. T. Conway, "Inductance calculations for noncoaxial coils using bessel functions," IEEE Transactions on Magnetics, vol. 43, no. 3, pp. 1023-1034, 2007.

[7] R. Ravaud, G. Lemarquand, S. Babic, V. Lemarquand, and C. Akyel, "Cylindrical magnets and coils: fields, forces, and inductances," IEEE Transactions on Magnetics, vol. 46, no. 9, pp. 3585-3590, 2010.

[8] R. Ravaud, G. Lemarquand, V. Lemarquand, S. Babic, and C. Akyel, "Mutual inductance and force exerted between thick coils," Progress in Electromagnetics Research, vol. 102, pp. 367380, 2010.

[9] A. Shiri and A. Shoulaie, "A new methodology for magnetic force calculations between planar spiral coils," Progress in Electromagnetics Research, vol. 95, pp. 39-57, 2009.

[10] R. Ravaud, G. Lemarquand, and V. Lemarquand, "Force and stiffness of passive magnetic bearings using permanent magnets. Part 1: axial magnetization," IEEE Transactions on Magnetics, vol. 45, no. 7, pp. 2996-3002, 2009.

[11] R. Ravaud, G. Lemarquand, and V. Lemarquand, "Force and stiffness of passive magnetic bearings using permanent magnets. part 2: radial magnetization," IEEE Transactions on Magnetics, vol. 45, no. 9, pp. 3334-3342, 2009.

[12] J. Coulomb and G. Meunier, "Finite element implementation of virtual work principle for magnetic or electric force and torque computation," IEEE Transactions on Magnetics, vol. 20, no. 5, pp. 1894-1896, 1984.

[13] A. Benhama, A. C. Williamson, and A. B. J. Reece, "Force and torque computation from 2_D and 3-D finite element field solutions," IEE Proceedings-Electric Power Applications Journal, vol. 146, no. 1, pp. 25-31, 1999.

[14] E. P. Furlani, "A formula for the levitation force between magnetic disks," IEEE Transactions on Magnetics, vol. 29, no. 6, pp. $4165-4169,1993$.

[15] E. P. Furlani, "Formulas for the force and torque of axial couplings," IEEE Transactions on Magnetics, vol. 29, no. 5, pp. 2295-2301, 1993.

[16] J. T. Conway, "Non coaxial force and inductance calculations for bitter coils and coils with uniform radial current distributions," in Proceedings of the International Conference on Applied Superconductivity and Electromagnetic Devices (ASEMD '11), pp. 61-64, Sydney, Australia, December 2011.

[17] Y. Ren, F. Wang, G. Kuang et al., "Mutual inductance and force calculations between coaxial bitter coils and superconducting coils with rectangular cross section," Journal of Superconductivity and Novel Magnetism, vol. 24, no. 5, pp. 1687-1691, 2011.
[18] M. Abramowitz and I. A. Stegun, Handbook of Mathematical Functions, Series 55, National Bureau of Standards Applied Mathematics, Washington, DC, USA, 1972.

[19] I. S. Gradshteyn and I. M. Ryzhik, Table of Integrals, Series and Products, Academic Press, New York, NY, USA, 1965. 


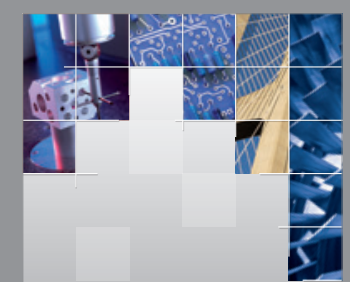

\section{Enfincering}
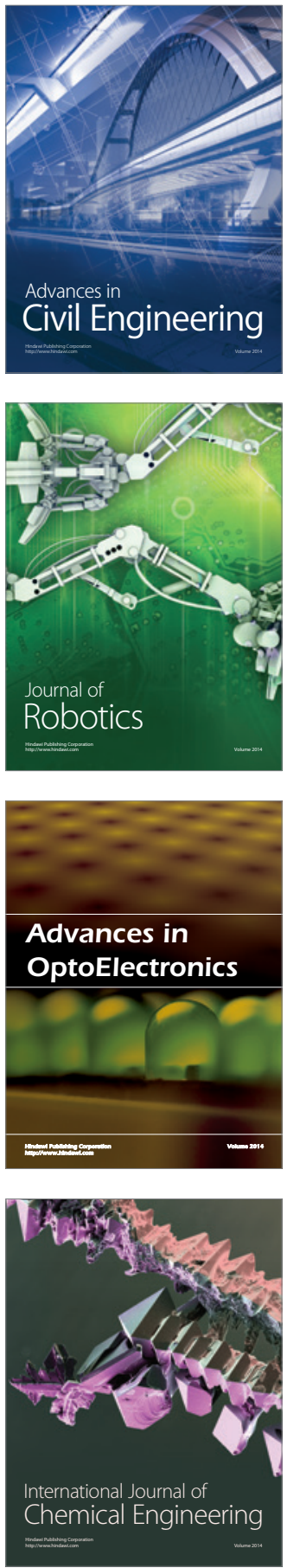

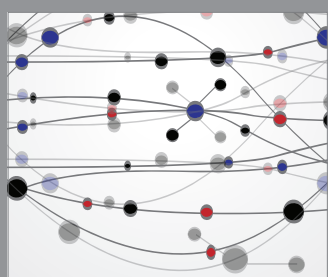

The Scientific World Journal

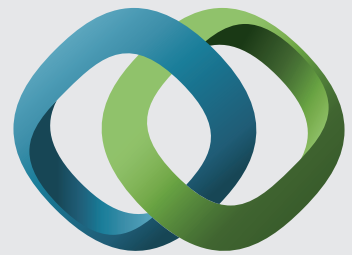

\section{Hindawi}

Submit your manuscripts at

http://www.hindawi.com
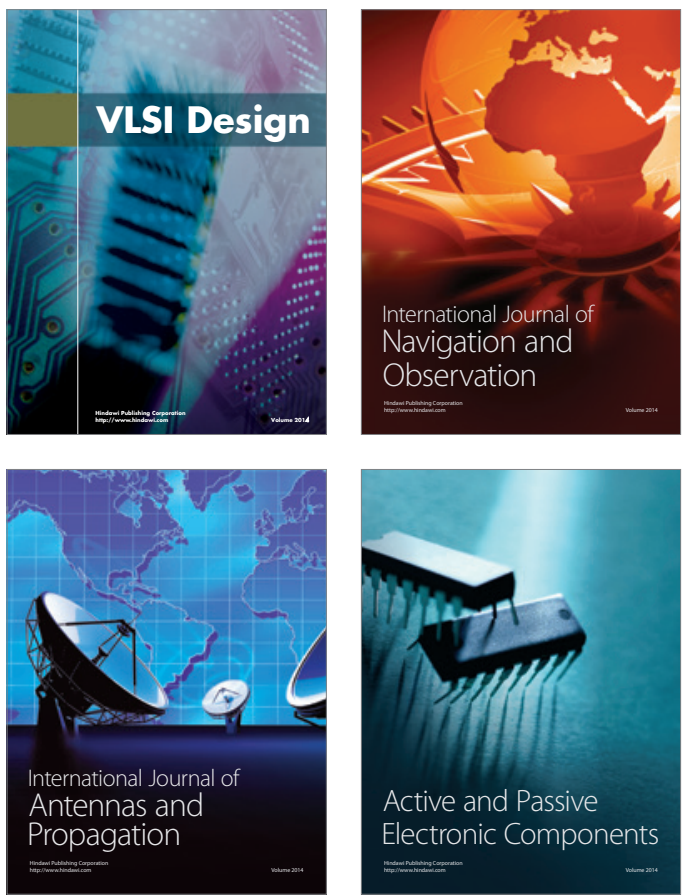
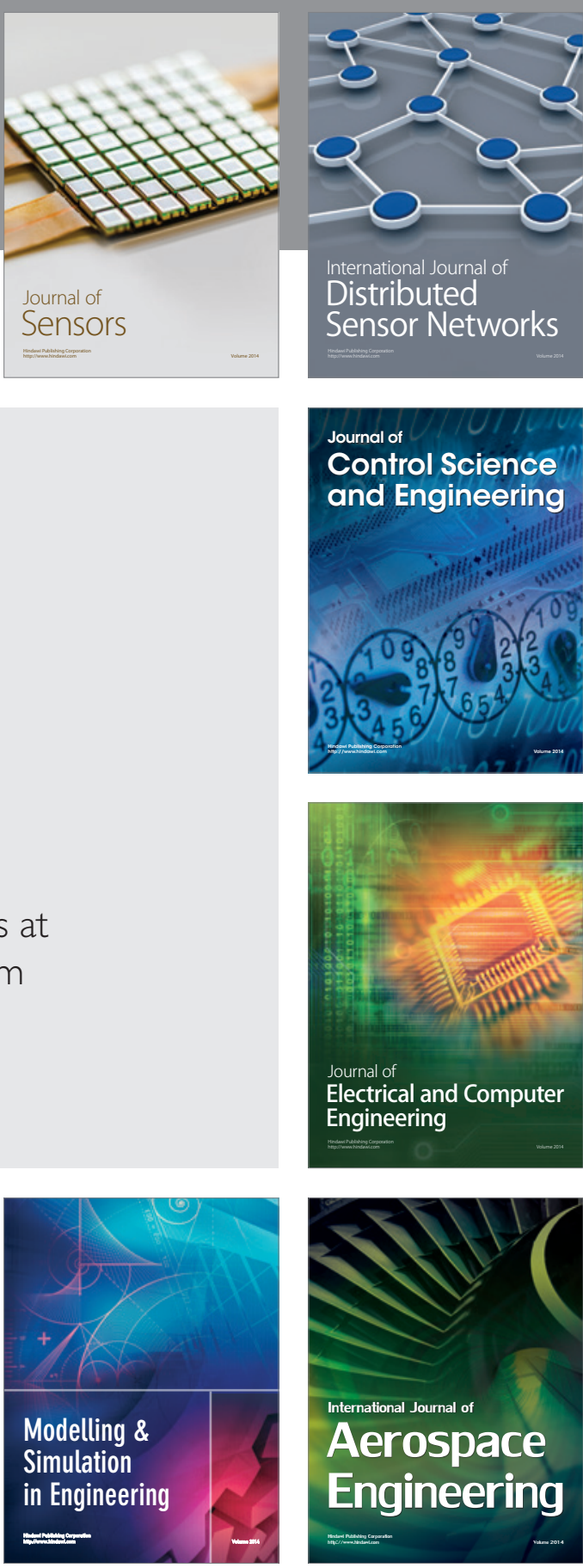

International Journal of

Distributed

Sensor Networks

Journal of

Control Science

and Engineering
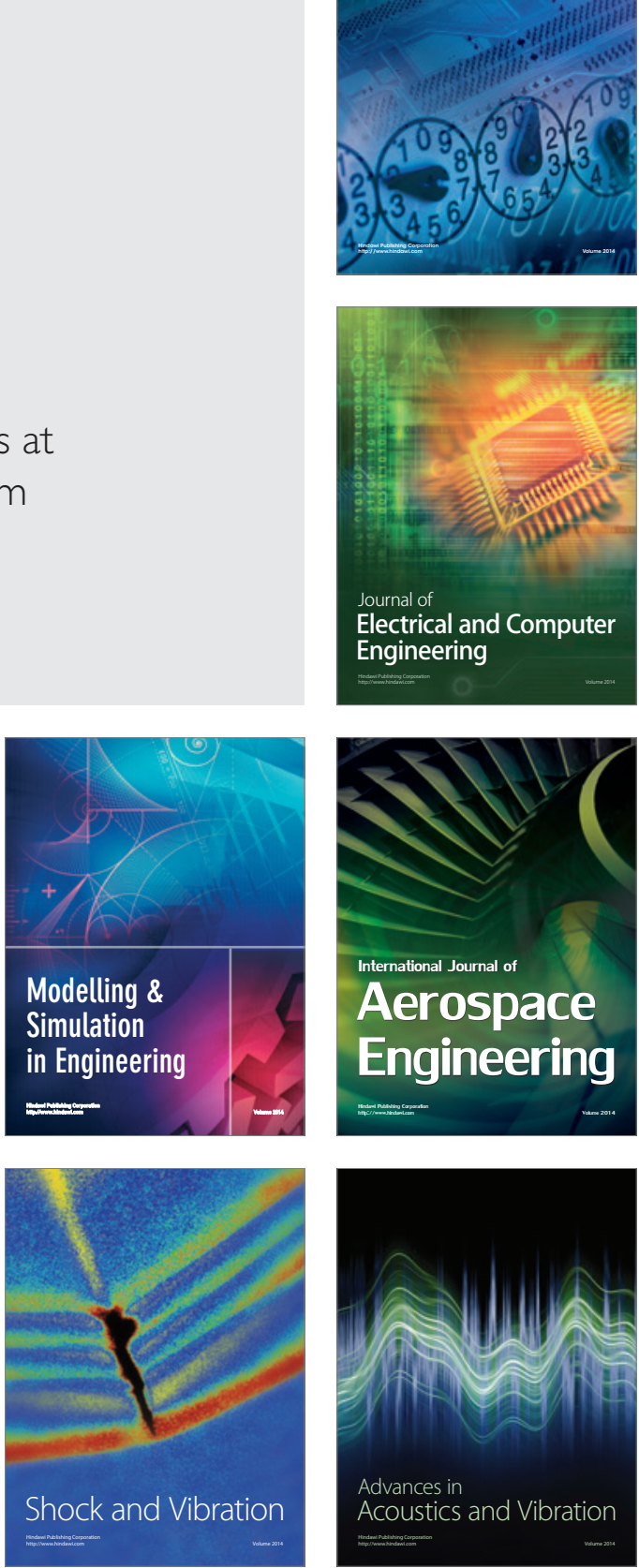